\title{
Medical Image of the Week: Coffee Bean and Whirlpool Signs
}

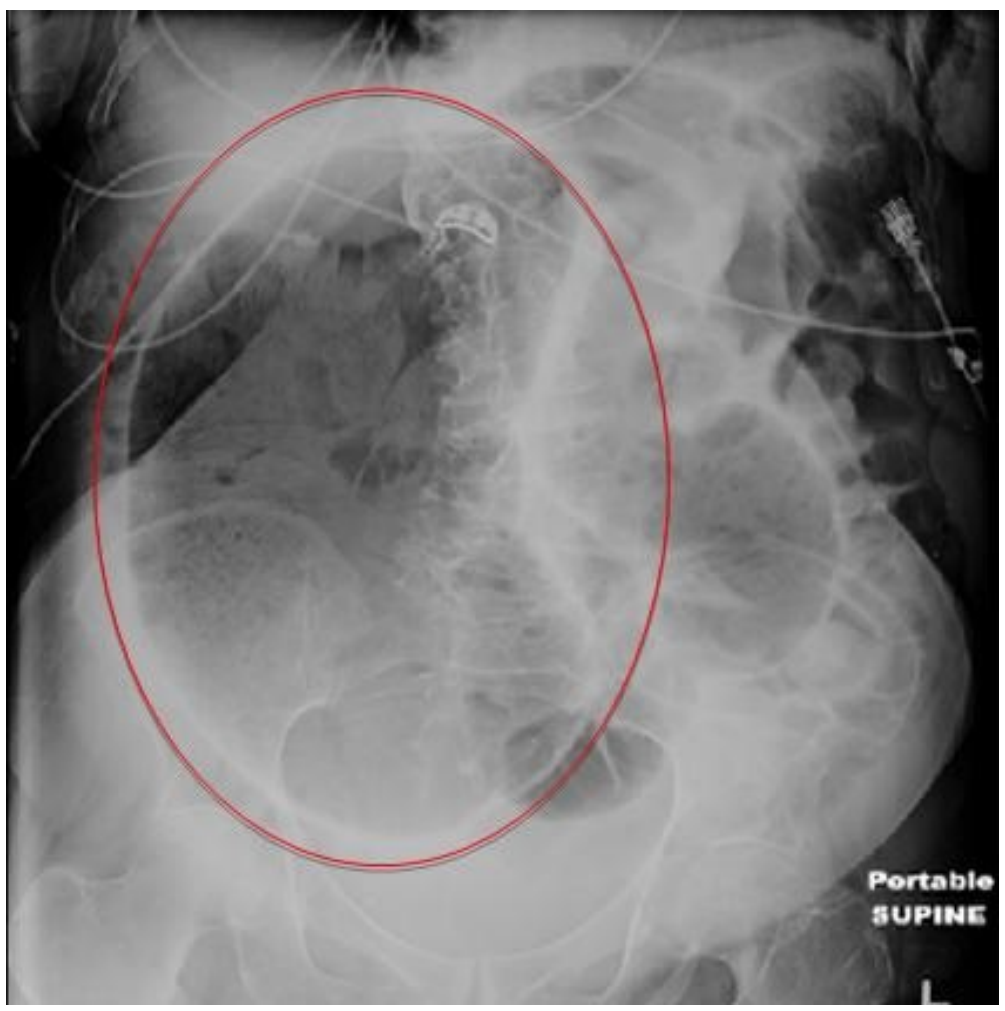

Figure 1. Supine abdominal $x$-ray demonstrating a large dilated loop of bowel and coffee bean sign (red circle).

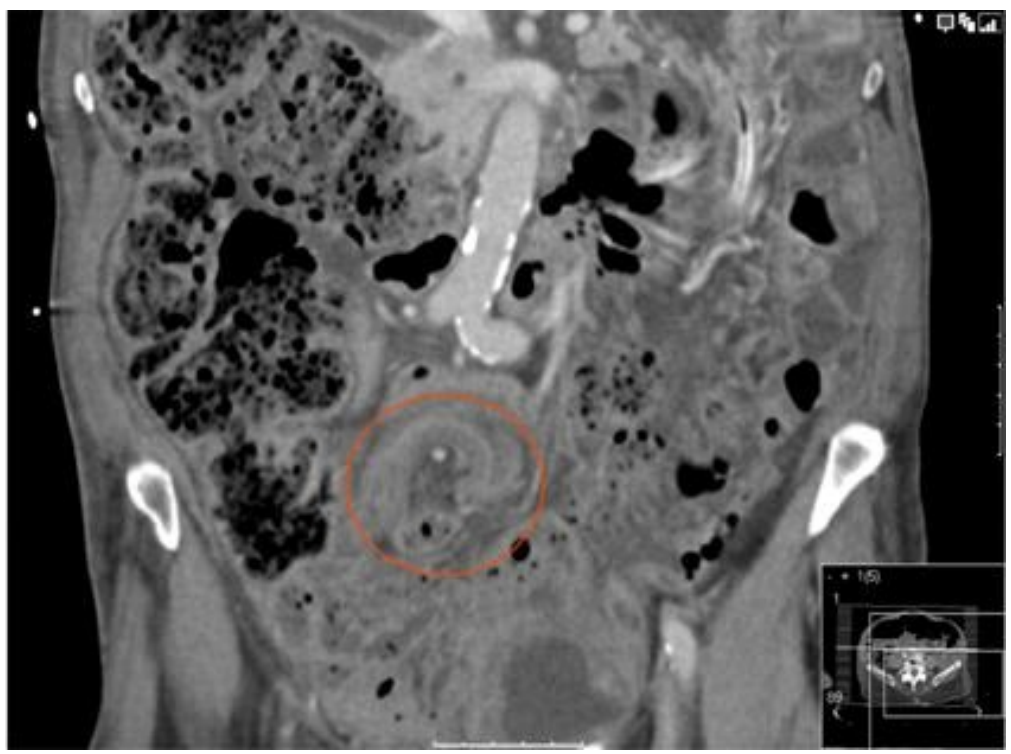

Figure 2. Contrast CT abdomen (coronal section) showing markedly dilated sigmoid loop with the swirling mesentery (whirlpool sign) (red circle). 
A 79-year-old woman with a history of Parkinson's disease presented with altered mental status, poor oral intake, and multiple episodes of nausea and vomiting. An abdominal x-ray demonstrated dilated loops of bowel and the coffee bean sign concerning for sigmoid volvulus (Figure 1). The coffee bean sign occurs when a thick "inner wall" represents the double wall thickness of opposed loops of bowel while the thinner outer walls due single thickness. A contrast CT abdomen showed dilated sigmoid loop and whirlpool sign confirming sigmoid volvulus (Figure 2). She underwent a total colectomy with ileorectal anastomosis and full recovery.

Brittany Bartolome $\mathrm{MS}^{1}$, Choua Thao $\mathrm{MD}^{2}$, Yaser Dawod $\mathrm{MD}^{2}$, and Carmen Luraschi $\mathrm{MD}^{3}$

${ }^{1}$ University of Nevada School of Medicine, Reno, NV USA

${ }^{2}$ Department of Internal Medicine, University of Nevada School of Medicine, Las Vegas, NV USA

${ }^{3}$ Division of Pulmonary and Critical Care, University of Nevada School of Medicine, Las Vegas, NV USA 